\title{
Calendrier pastoral et carte de transhumance des éleveurs exploitant les ressources pastorales de la commune de Djidja au Sud Bénin
}

\author{
Maximilien Azalou ${ }^{1}$ * Alassan Assani Seidou ${ }^{1}$ \\ Brice Gérard Comlan Assogba ${ }^{1}$ Josias Steve Adjassin ${ }^{1}$ \\ Hilaire Sorébou Sanni Worogo ${ }^{1}$ Mohamed Nasser Baco ${ }^{2}$ \\ Ibrahim Alkoiret Traoré ${ }^{1}$
}

\begin{abstract}
Mots-clés
Bovin, système d'élevage, calendrier, transhumance, résidu de récolte, pastoralisme, Bénin
\end{abstract}

Submitted: 21 May 2018

Accepted: 9 April 2019

Published: 20 May 2019

DOI: $10.19182 /$ remvt.31727

\section{Résumé}

Djidja est l'une des plus grandes communes productrices agricoles du département du Zou au sud du Bénin. De par ses ressources fourragères et hydriques, elle est devenue une destination des éleveurs transhumants. Les mouvements des éleveurs transhumants continuent de s'étendre, de même que les séjours dans cette zone d'accueil s'allongent. L'étude a eu pour objectif global d'élaborer le calendrier pastoral et la carte de transhumance des éleveurs fréquentant cette commune. Des entretiens semi-structurés ont été conduits auprès de 300 acteurs de la transhumance. L'enquête a montré que la transhumance dans cette commune était surtout due à la recherche de ressources fourragères et hydriques (78,7\%). Sept périodes (Seeto, Nduungu Mawdo, Nduungu Pamarel, Jahol, Djaamdè, Dabuundè et Cheedu) ont été identifiées dans le calendrier des transhumants fréquentant cette zone avec une particularité de deux périodes de Nduungu (saison des pluies). Cette particularité est liée aux données climatiques de la zone qui comprend quatre saisons, dont deux pluvieuses et deux sèches. Le calendrier pastoral, l'itinéraire suivi et les temps de séjour dépendaient de la disponibilité des ressources pastorales des zones d'attache, de transit et d'accueil. Ainsi, la bonne connaissance des pistes empruntées, des points d'entrées et de sorties, et des périodes d'accueil des éleveurs transhumants serviront d'outils aux décideurs en matière de gestion durable de la transhumance et des ressources pastorales au sud du Bénin.

- Comment citer cet article : Azalou M., Assani Seidou A., Assogba B.G.C.,Adjassin J.S., Sanni Worogo H.S., Baco M.N., Alkoiret Traoré I., 2019. Pastoral calendar and transhumance map of herders using pastoral resources in Djidja Commune in Southern Benin. Rev. Elev. Med. Vet. Pays Trop., 72 (1): 3-11, doi: $10.19182 /$ remvt.31727

\section{INTRODUCTION}

Le système pastoral, mobile et extensif est basé sur la transhumance. Les animaux et les hommes des pays du sahel se déplacent chaque année vers les pays côtiers à la recherche de ressources pastorales. Les critères d'appréciation d'un pâturage sont la quantité et la qualité (plus ou moins vert, ligneux, riche en graines...). Ainsi, la diversité

\footnotetext{
1. Laboratoire d'écologie, de santé et de production animales (LESPA), Faculté d'agronomie, Université de Parakou, BP 123, Parakou, Bénin.

2. Laboratoire société-environnement (LaSEn), Faculté d'agronomie, Université de Parakou, Bénin.

* Auteur pour la correspondance

Tél. : +22997516463 ; email : maxazaloux@yahoo.fr
}

d'un pâturage, son évolution dans le temps et dans l'espace, son accessibilité aux différentes périodes sont autant d'éléments qui permettent à l'éleveur de décider des lieux de pâture et des dates de déplacements (Schönegg et al., 2006). Cette mobilité saisonnière constitue une adaptation du système au changement climatique dans les zones sahéliennes, soudaniennes et soudano-guinéennes (Duteurtre et al., 2002). La transhumance est également une forme de valorisation des grands espaces. Cela peut engendrer des problèmes dans le contexte actuel de forte pression foncière, de changement climatique et de croissance démographique (Harchies et al., 2007). Cependant, certains éleveurs transhumants prennent des dispositions pour prévenir les crises liées à l'exploitation des ressources pastorales (Marty et al., 2006).

Au Sud Bénin, l'élevage bovin contribue à $20 \%$ au revenu des éleveurs. Il est très développé autour des villes et des villages des zones lacustres des communes de Sô Ava, Dangbo et Abomey-Calavi (Floquet et Nansi, 
2005). Pour assurer l'alimentation des troupeaux bovins, les éleveurs ont essentiellement recours à l'utilisation des pâturages naturels. Les écarts importants de la disponibilité fourragère dans les régions sèches imposent au bétail d'être mobile, de façon à aller chercher les ressources là où elles se trouvent (Toutain et al., 2012). Pour la plupart des éleveurs, la transhumance est une stratégie d'adaptation et d'exploitation des ressources pastorales face à la baisse des précipitations et aux crises fourragères. Elle permet aux éleveurs d'exploiter les complémentarités intrazonales ou interzonales (Kiema et al., 2014). Ainsi, la mobi-

lite spatio-temporelle a été développée par les éleveurs, leur permettan de suivre la qualité des fourrages encore disponibles sur les pâturages afin d'assurer les productions du troupeau (de Jode, 2010 ; Djenontin et al., 2012). Le problème de l'utilisation des aires de pâture par le bétail devient de plus en plus complexe en raison de l'avancée des cultures sur les zones de parcours, de l'installation des champs autour de points d'eau pastoraux et de la diversification des activités des producteurs assurant ainsi une amélioration de leurs revenus (Leclerc et Sy, 2011). En ce qui concerne la mobilité pastorale, les éleveurs transhumants restent très flexibles dans le choix de leurs itinéraires, qui serait déterminant pour la survie des troupeaux.

La commune de Djidja dispose par endroits d'îlots de forêts donnant l'aspect d'une végétation arborée ou arbustive, et de deux forêts classées, entretenues et complétées par des plantations de teck à Dan et Setto (Guidibi et Akomagni, 2006). De par ses ressources, elle est devenue une destination des transhumants nationaux et étrangers, malgré les conflits qui opposent les éleveurs aux agriculteurs. Trois types de troupeaux transhumants ont été identifiés dans la zone d'étude : les transhumants transfrontaliers (type 1), les transhumants intercommunaux (type 2) et les transhumants semi-sédentaires (type 3) (Azalou, 2016). La divagation des animaux dans les champs des agriculteurs, les itinéraires, et les périodes de départ et de retour, fixés par la loi pour la transhumance non adaptée aux réalités du terrain, sont les causes fondamentales des conflits (Assani, 2017 ; Hiya Maidaya et al., 2016 ; Lesse, 2016). Le présent article a eu pour objectif de contribuer à une meilleure connaissance des itinéraires et des périodes de fréquentation des éleveurs trans-

\section{MATERIEL ET METHODES}

\section{Milieu d'étude}

L'étude a été réalisée dans la commune de Djidja, au sud-ouest du Bénin (figure 1) dans le département du Zou, à environ 180 mètres d'altitude, entre $7^{\circ} 10^{\prime}$ et $7^{\circ} 31^{\prime} \mathrm{N}$, et $1^{\circ} 39^{\prime}$ et $1^{\circ} 1^{\prime}$ ' E. Elle s'étend sur 2184 kilomètres carrés (41,7\% de la superficie du département) (Guidibi et Akomagni, 2006). Son climat est de type tropical humide, caractérisé par quatre saisons : une grande saison des pluies de mi-mars à mi-juillet, une petite saison sèche de mi-juillet à début septembre, une petite saison pluvieuse de septembre à mi-novembre, et une grande saison sèche de mi-novembre à mi-mars. A Djidja, les pluies annuelles atteignent 1100-1200 mm. En année moyenne la saison pluvieuse commence en mars-avril avec des précipitations mensuelles atteignant $90 \mathrm{~mm}$ (Vissin, 2015). La température moyenne est de $28,5^{\circ} \mathrm{C}$; les maxima sont de l'ordre de $36-37{ }^{\circ} \mathrm{C}$ entre février et mars, et les minima de $22-25^{\circ} \mathrm{C}$ entre juillet et novembre.

\section{Méthodes}

Les entretiens avec les éleveurs transhumants, agroéleveurs et/ou éleveurs sédentaires, les personnes-ressources, et les observations sur le terrain ont constitué les approches utilisées. Les entretiens ont été menés entre mars 2015 et novembre 2016. Afin de comprendre les périodes et raisons d'entrée des éleveurs à Djidja et les itinéraires suivis, la méthode d'échantillonnage aléatoire a été utilisée. Neuf villages ont été retenus pour élaborer le calendrier thématique dans cinq arrondissements de la commune. Le choix des villages a été fait avec les responsables des éleveurs de Djidja, Setto, Dan et Agouna, et les responsables en charge de la production animale à Djidja. Les critères de ce choix ont été basés essentiellement sur l'accueil des éleveurs transhumants, les marchés de bétail et la concentration des élevages de bovins dans ces villages qui abritaient les éleveurs même après la période de grande transhumance. Un groupe de discussion a été organisé au niveau de chaque village. Tous les éleveurs transhumants rencontrés (112) ont été retenus pour constituer l'échantillonnage. Ils représentaient $27 \%$ de l'effectif des transhumants totaux (421) selon les services de l'élevage. Les données ont été collectées sur la base d'entretiens semi-structurés et structurés. La méthode d'échantillonnage aléatoire simple a été appliquée (Ardilly, 2006). Un taux de sondage de $92,4 \%$ a permis de déterminer la taille de l'échantillon (n) avec la formule de Dagnelie (1998) :

$$
\frac{U_{1-\alpha / 2}{ }^{2} \times P i(1-P i)}{d^{2}}
$$

où $U_{1-\alpha / 2}$ représente la valeur de la variable aléatoire normale pour une valeur de la probabilité pour un seuil de signification $\alpha=0,05, U^{2}{ }_{1}$. $\alpha / 2=3,84$ et $d=0,03$ la marge d'erreur fixée en tenant compte des travaux antérieurs sur les acteurs de transhumance (Assani, 2017 ; Lesse, 2016). Ainsi, le nombre des différents acteurs à enquêter était égal à 300 , et ils étaient proportionnellement répartis entre les cinq arrondissements retenus (Agouna, Dan, Djidja, Monsourou, et Setto) (tableau I).

\section{Calendrier pastoral}

L'élaboration du calendrier pastoral s'est inspirée des travaux de Kagoné et al. (2006) au Burkina Faso, et de Djenontin et al. (2012) au Bénin. Les éleveurs de bovins enquêtés ont été réunis par village et à l'aide d'un guide d'entretien nous avons analysé avec eux les questions sur les différentes périodes de l'année et leurs durées, les périodes de pic

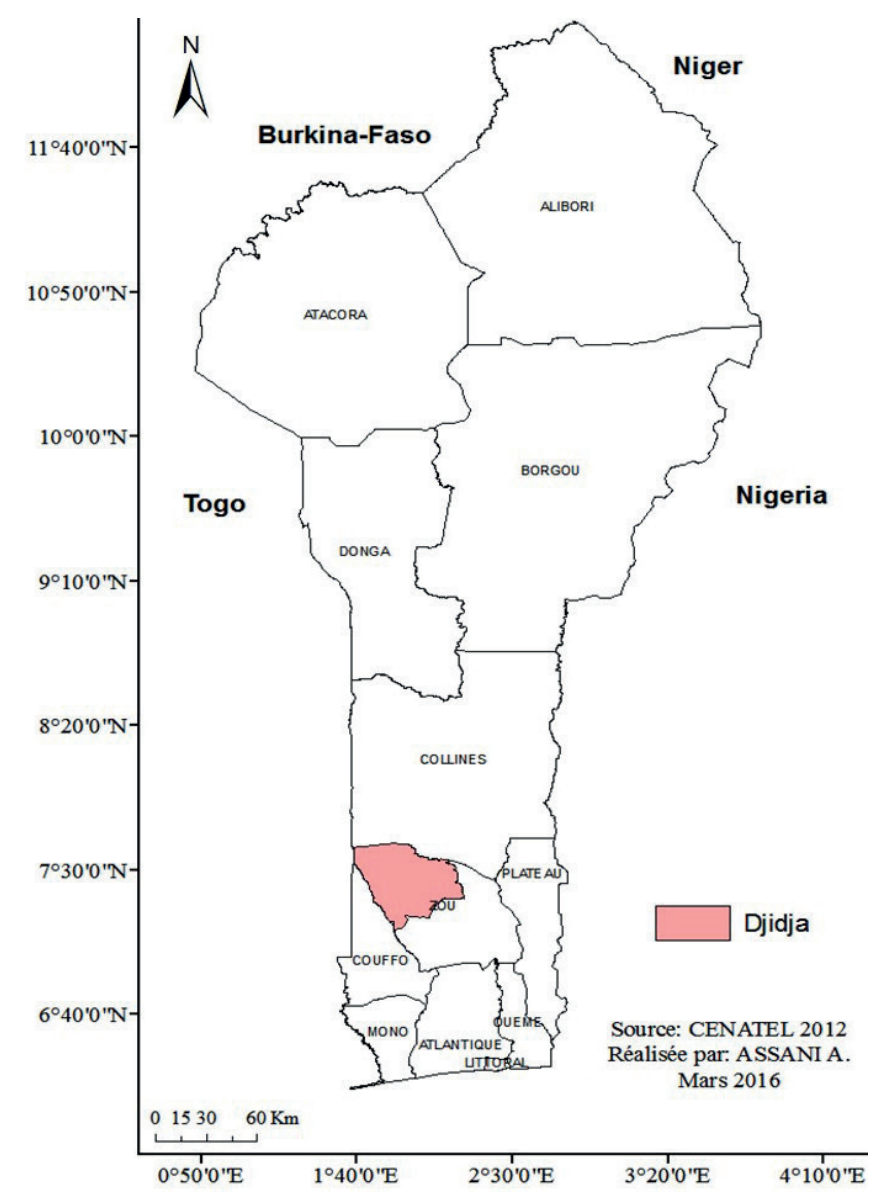

Figure 1 : localisation de la commune de Djidja au Sud Bénin. 


\section{Tableau I}

Acteurs de la transhumance et leur répartition dans les villages de la commune de Djidja au Bénin (mars 2015 à novembre 2016)

\begin{tabular}{|c|c|c|}
\hline Acteurs & Nb. & \\
\hline Eleveur & 112 & \\
\hline Agriculteur & 91 & \\
\hline Chasseur & 12 & \\
\hline Pêcheur & 11 & \\
\hline Garso & 15 & \\
\hline Responsable d'association d'éleveur & Irs 15 & \\
\hline Agent forestier & 5 & \\
\hline $\begin{array}{l}\text { Services déconcentrés de l'Etat } \\
\left(\text { mairie, Carder }^{1}, \text { SCDA }^{2}\right)\end{array}$ & 14 & \\
\hline Organismes non gouvernementaux & 9 & \\
\hline Chef coutumier (chef de village) & 16 & \\
\hline Total & 300 & \\
\hline Arrondissements & Villages & Nb. d'acteurs \\
\hline \multirow[t]{2}{*}{ Agouna } & Sankpiti & 33 \\
\hline & Denou & 34 \\
\hline Dan & Dan & 32 \\
\hline \multirow[t]{2}{*}{ Djidja } & Sovlègni & 36 \\
\hline & Zinkanmè & 38 \\
\hline \multirow[t]{2}{*}{ Monsourou } & Kaka Tèhou & 31 \\
\hline & Monsourou & 33 \\
\hline \multirow[t]{2}{*}{ Setto } & Setto & 33 \\
\hline & Gbadagba & 30 \\
\hline
\end{tabular}

${ }^{1}$ Centres d'action régionale pour le développement rural $;{ }^{2}$ Secteur communal pour le développement agricole

de fréquentation de la commune au cours de l'année par les éleveurs transhumants, et les activités des éleveurs locaux et étrangers au cours de ces périodes. Ensuite, du point de vue thématique, les périodes d'activités pastorales et agricoles ont été précisées, ainsi que les positions des troupeaux au cours de l'année. Nous avons pu alors dessiner sur une feuille avec les différents groupes de discussion une bande représentant l'année, et marquer les périodes et les événements. Un jeu de cailloux a permis de déterminer l'importance relative de chaque événement au cours de chaque période (figure 2). Il a été demandé aux groupes de personnes d'estimer la proportion d'animaux présents ainsi que l'importance des activités pastorales dans cette commune pendant chaque période du calendrier pastoral.

Pour traiter ces informations, les données collectées ont été soumises à l'analyse de fiabilité pour être appréciées par des personnes-ressources. Ces dernières ont été retenues parmi les acteurs de gestion de la transhumance à Djidja (éleveurs, bouviers et autorités locales). L'analyse d'intégrité a été réalisée après la classification des données sur la base de références adaptées au terroir des éleveurs et à l'élevage bovin (Bardin, 1998 ; Apostolidis, 2006). L'identification et la classification des données ont été assurées par l'analyse du contenu.

\section{Carte de transhumance de la commune de Djidja}

Pour la collecte des données d'enquête, deux méthodes ont été adoptées (Assani, 2017) :

- une méthode qualitative qui a consisté en une collecte d'informations à travers des entretiens informels (individuels ou en groupe)

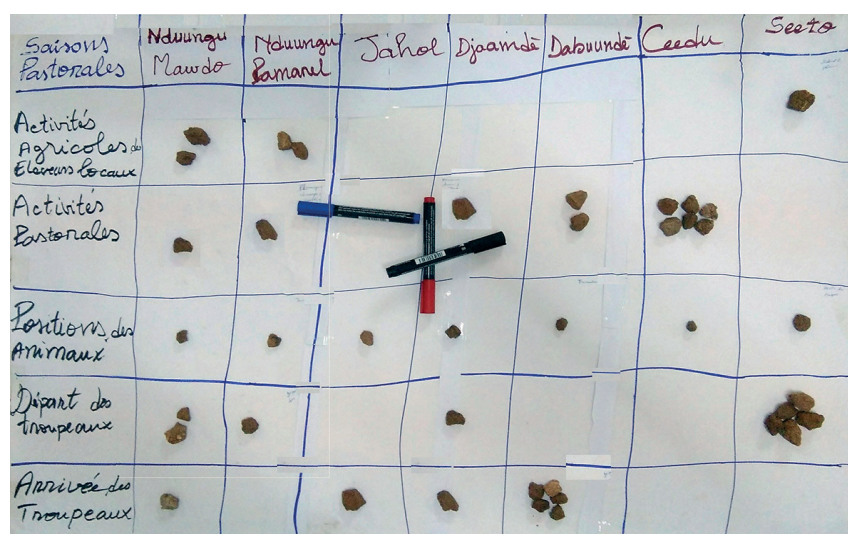

Figure 2 : jeu de cailloux pour l'élaboration du calendrier pastoral dans la commune de Djidja (Sud Bénin).

avec les acteurs directs de la transhumance et de l'élevage dans la zone d'étude. Des observations participantes à travers des enquêtes de terrain ont également été faites. Cette méthode a permis de vivre les réalités des éleveurs enquêtés et d'avoir un accès privilégié aux informations inaccessibles ;

- une méthode quantitative qui a été menée par des observations standardisées, en l'occurrence à l'aide d'un questionnaire adressé aux transhumants étrangers et locaux des neuf villages. Les éleveurs et les agroéleveurs sont des personnes qui habitent le village depuis plusieurs années et maîtrisent les axes de transhumance de la zone. La spatialisation des éléments structurants des axes de transhumance (terroirs d'attache, zone de transit, zone d'accueil) a été réalisée avec un système de géolocalisation (GPS) de type Garmin (Etrex Vista) et le logiciel SIG (ArcView). Les relevés des points de repère sur le terrain ont été complétés par les données d'enquêtes. Enfin, les itinéraires de transhumance ont été obtenus en superposant aux données de l'étude un fond de carte topographique IGN de 1992.

\section{RESULTATS}

\section{Calendrier pastoral des éleveurs fréquentant Djidja}

La décision de partir en transhumance relevait de l'éleveur propriétaire. Ainsi, les périodes de départ ou de retour des animaux en transhumance variaient en fonction de la pluviosité de l'année en cours, de l'état des pâturages et des points d'eau, mais aussi des patrouilles forestières. La figure 3 présente la succession des périodes dans le calendrier pastoral des éleveurs à Djidja. La conduite du troupeau

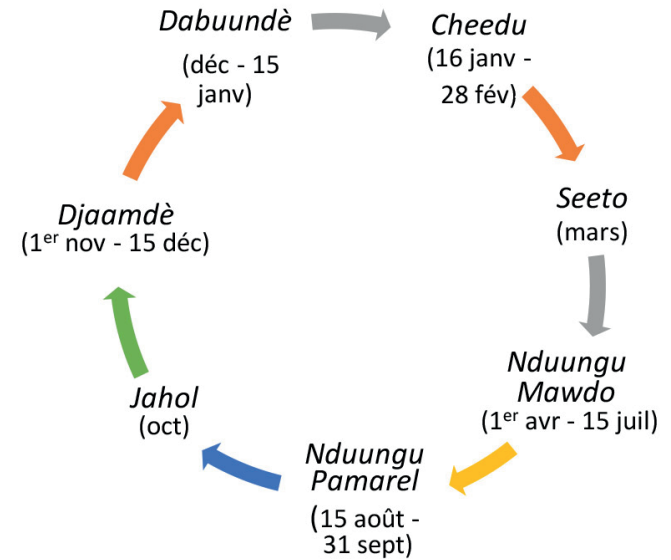

Figure 3 : succession des périodes dans le calendrier pastoral des éleveurs transhumants dans la commune de Djidja (Sud Bénin). 
s'appuyait sur les sept périodes du calendrier agropastoral peul : Dabuundè, Cheedu, Seeto, Nduungu Mawdo, Nduungu Pamarel, Jahol, et Djaamdè. Le calendrier agropastoral en sept périodes rend compte des différentes interactions synergiques ou conflictuelles qui existent à l'échelle des terroirs à différentes périodes de l'année entre l'agriculture et l'élevage. Les activités agricoles et d'élevage occupent une grande partie de l'année, bien que certains mois ou périodes pastorales soient plus chargés que d'autres.

Dabuundè (saison sèche froide ou harmattan) intervient après la récolte des céréales (Djaamdè) à Djidja. Le bétail des agriculteurs et celui des éleveurs sont en général bien alimentés à cette période. Elle correspond au départ des troupeaux pour la grande transhumance et au début de l'arrivée des transhumants étrangers.

Cheedu (saison sèche chaude) s'étend de janvier jusqu'au début des pluies. Les animaux connaissent une période de pénurie alimentaire liée à l'épuisement des résidus de culture et à l'abondance des troupeaux transhumants dans la commune. L'exploitation de ligneux fourragers comme Pterocarpus erinaceus, Terminalia glaucescens, Khaya senegalensis et Afzelia africana, le pâturage des herbacées le long des cours d'eau et l'abreuvement dans les retenues d'eau, les fleuves Zou et Couffo, sont les activités pastorales pendant cette période.

Seeto (début des pluies ou période de transition sèche pluvieuse) est marquée par le retour des troupeaux de la grande transhumance et la régénération des pâturages suite aux premières pluies à Djidja. Pour assurer l'alimentation des animaux, certains éleveurs, par mesure de sécurité, conduisent leur troupeau dans les zones immergées, pendant cette période où les premières repousses de graminées servent d'aliment pour le bétail.

Nduungu Mawdo (en peul signifie grande saison des pluies) intéresse, dans la zone d'étude, une partie des troupeaux appelés « bœufs de trait et noyau laitier » qui s'adaptent aux besoins alimentaires et sociaux de la famille de l'éleveur ainsi qu'aux ressources pastorales disponibles. Ces petits troupeaux bovins sont sédentaires et bénéficient de la végétation spontanée d'herbacées. L'objectif de l'éleveur est de s'éloigner des zones de culture afin d'éviter la dégradation des champs par les animaux. Le nombre d'animaux dans cette commune est réduit considérablement compte tenu des activités agricoles.

Nduungu Pamarel (petite saison des pluies) est similaire à la précédente. Les éleveurs ayant effectué une petite transhumance sont toujours éloignés des zones de cultures. La pluviosité ici est faible par rapport à la première. Les pâturages d'herbe fraîche autour des campements sont abondants et de bonne qualité.

Jahol (baisse des précipitations) annonce la maturité des céréales et le début des récoltes. Les éleveurs transhumants descendent progressivement rejoindre les zones d'attache lorsque les résidus de récoltes sont disponibles.

Djaamdè (saison des récoltes des cultures) permet au bétail de profiter au maximum des repousses spontanées et des pâturages postculturaux. Les fanes de niébé, d'arachide sont valorisées au début de la période ; les pâturages en lignification sont les plus utilisés ; les troupeaux restent dans leur zone d'attache.

L'accès aux ressources pastorales diffère d'une ressource à une autre. L'enquête a montré que les modes d'accès aux ressources fourragères et hydriques variaient en fonction des lieux de pâturage et des points d'abreuvement. L'accès des troupeaux bovins en transhumance à l'eau d'abreuvement pouvait être libre (54\%) ou payant (46\%). Cette situation indique la compétition qu'il y a autour de l'accès à l'eau par rapport au fourrage. En effet, l'accès à l'eau de surface des fleuves Zou et Couffo est généralement libre.

\section{Calendrier pastoral et diagramme climatique de la zone d'étude}

La superposition du calendrier pastoral au diagramme climatique a permis de relever des traits pratiques de gestion du temps et de l'espace par les éleveurs transhumants (figure 4). Ainsi, la période préhumide correspondait à Seeto, la période humide à Nduungu Mawdo, N. Pamarel, Jahol et Djaamdè, et la période posthumide à Dabuundè et Cheedu.

Le tableau II présente le calendrier pastoral et les activités agricoles des éleveurs locaux et étrangers durant chaque période.

\section{Carte de transhumance des éleveurs fréquentant Djidja}

Les différents arrondissements et villages qui accueillaient les transhumants décrivaient les axes de transhumance. Les transhumants nationaux et étrangers empruntaient six axes à Djidja. Ils constituaient les principaux axes empruntés par les éleveurs étrangers ; $60 \%$ faisaient leur entrée par l'arrondissement de Setto. Sur la base de quelques points de passage indiqués, on peut définir les axes empruntés par les transhumants (figure 5).

\section{Raisons du choix des itinéraires}

Le choix de l'itinéraire de mouvement et du campement de nuit était fonction de la disponibilité en eau, en pâturage, en résidus de récolte, de l'existence de marchés, de la sécurité et de l'accueil des populations des zones traversées. Ces contraintes étaient d'importance variable suivant les éleveurs. Tous les éleveurs (100\%) enquêtés basaient le choix de l'itinéraire et du campement de nuit sur la disponibilité en eau. Concernant les autres facteurs, les avis étaient diversifiés (figure 6) :

- le pâturage. Il guide le choix des itinéraires de mouvements ainsi que le lieu de campement (42,6\% des éleveurs). Il constitue un élément déterminant dans les mouvements des Foulani et surtout de ceux qui partent loin en transhumance. Par exemple le problème de pâturage ne se pose pas aux éleveurs résidents car la plupart sont aussi agriculteurs ;

- la sécurité, perçue en termes de maladies des bêtes et des hommes, et de vols d'animaux (21,2\% des éleveurs);

- les résidus de récoltes, très appétés par les animaux (17,9\% des éleveurs). Les résidus de récoltes ne sont disponibles que pendant la saison sèche fraîche ;

- les marchés (10,3\% des éleveurs). Au cours du trajet, il y a de petites dépenses (achat de sel, produits vétérinaires, provisions, etc.) à effectuer dans les marchés des villages où des échanges d'informations ont souvent lieu. Les marchés des communes environnantes, dont Bohicon et Abomey accueillent les animaux en transhumance pour la vente ;

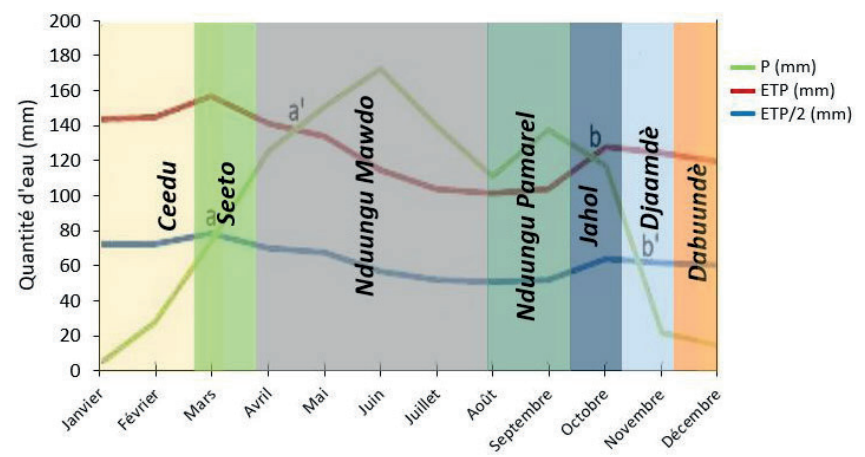

Figure 4 : superposition du calendrier pastoral au diagramme climatique dans la commune de Djidja (Sud Bénin). P : pluie ; ETP : évapotranspiration ; ETP/2 : évapotranspiration/2 


\section{Tableau II}

Calendrier pastoral des éleveurs dans la commune de Djidja au Bénin (mars 2015 à novembre 2016)

\begin{tabular}{|c|c|c|c|c|c|c|c|}
\hline $\begin{array}{l}\text { Périodes } \\
\text { peules }\end{array}$ & Dabuundè & Cheedu & Seeto & $\begin{array}{l}\text { Nduungu } \\
\text { Mawdo }\end{array}$ & $\begin{array}{l}\text { Nduungu } \\
\text { Pamarel }\end{array}$ & Jahol & Djaamdè \\
\hline & $\begin{array}{l}\text { Saison } \\
\text { sèche froide }\end{array}$ & $\begin{array}{l}\text { Saison } \\
\text { sèche chaude }\end{array}$ & $\begin{array}{l}\text { Début } \\
\text { des pluies }\end{array}$ & $\begin{array}{l}\text { Grande saison } \\
\text { des pluies }\end{array}$ & $\begin{array}{l}\text { Petite saison } \\
\text { des pluies }\end{array}$ & $\begin{array}{l}\text { Baisse } \\
\text { des pluies }\end{array}$ & $\begin{array}{l}\text { Fin } \\
\text { des pluies }\end{array}$ \\
\hline & Déc. - mi-janv. & Mi-janv. - fév. & Mars & Avr. - mi-juil. & Mi-août - sept. & Oct. & Nov. - mi déc. \\
\hline $\begin{array}{l}\text { Position des } \\
\text { Animaux }\end{array}$ & $\begin{array}{l}\text { Départ de } \\
\text { la grande } \\
\text { transhumance }\end{array}$ & $\begin{array}{l}\text { Zones d'accueil } \\
\text { de la grande } \\
\text { transhumance }\end{array}$ & $\begin{array}{l}\text { Retour de } \\
\text { la grande } \\
\text { transhumance }\end{array}$ & \multicolumn{3}{|c|}{ Petite transhumance } & $\begin{array}{l}\text { Zone d'attache / } \\
\text { arrivée précoce }\end{array}$ \\
\hline $\begin{array}{l}\text { Activités } \\
\text { pastorales }\end{array}$ & $\begin{array}{l}\text { - Vaine pâture } \\
\text { dans les champs } \\
\text { de céréales après } \\
\text { les récoltes (maïs, } \\
\text { niébé, arachide) } \\
\text { - Abreuvement } \\
\text { dans les cours } \\
\text { d'eau et retenues } \\
\text { d'eau non taries }\end{array}$ & $\begin{array}{l}\text { - Pâturage sur } \\
\text { résidus après les } \\
\text { récoltes tardives } \\
\text { - Pâturage } \\
\text { de nouvelles } \\
\text { repousses } \\
\text { de ligneux } \\
\text { sur parcours } \\
\text { (Afzelia africana, } \\
\text { Pterocarpus } \\
\text { erinaceus...) et } \\
\text { des herbes le } \\
\text { long des bas- } \\
\text { fonds }\end{array}$ & $\begin{array}{l}\text { - Pâturage } \\
\text { d'herbe fraîche } \\
\text { de graminées } \\
\text { précoces } \\
\text { - Abreuvement } \\
\text { au niveau des } \\
\text { fleuves Zou, } \\
\text { Couffo et des } \\
\text { cours d'eau }\end{array}$ & $\begin{array}{l}\text { - Pâturage } \\
\text { d'herbe fraîche } \\
\text { autour des } \\
\text { campements, } \\
\text { jachères } \\
\text { - Abreuvement } \\
\text { dans les cours } \\
\text { d'eau }\end{array}$ & $\begin{array}{l}\text { - Pâturage } \\
\text { d'herbe fraîche } \\
\text { abondante autour } \\
\text { des campements, } \\
\text { jachères } \\
\text { - Abreuvement } \\
\text { dans les cours } \\
\text { d'eau et retenues } \\
\text { d'eau }\end{array}$ & $\begin{array}{l}\text { - Pâturage } \\
\text { d'herbe fraîche } \\
\text { en fin de } \\
\text { croissance } \\
\text { - Abreuvement } \\
\text { dans les cours } \\
\text { d'eau }\end{array}$ & $\begin{array}{l}\text { - Pâturage } \\
\text { d'herbes moins } \\
\text { lignifiées } \\
\text { - Abreuvement } \\
\text { dans les cours } \\
\text { d'eau }\end{array}$ \\
\hline $\begin{array}{l}\text { Activités } \\
\text { agricoles } \\
\text { des éleveurs } \\
\text { locaux }\end{array}$ & $\begin{array}{l}\text { Récolte du maïs, } \\
\text { niébé, sorgho }\end{array}$ & $\begin{array}{l}\text { - Feu de brousse } \\
\text { - Récolte du } \\
\text { sorgho en début } \\
\text { de saison, } \\
\text { plantation } \\
\text { d'igname }\end{array}$ & $\begin{array}{l}\text { - Préparation } \\
\text { des champs : } \\
\text { défrichement, } \\
\text { labour + semis } \\
\text { du maïs, sorgho, } \\
\text { arachide }\end{array}$ & $\begin{array}{l}\text { - Travaux } \\
\text { d'entretien } \\
\text { (sarclo-buttage) } \\
\text { - Récolte et } \\
\text { stockage du } \\
\text { maïs, d'arachide, } \\
\text { haricots } \\
\text { - Préparation des } \\
\text { champs et mise } \\
\text { en place des } \\
\text { champs de maïs, } \\
\text { manioc en fin de } \\
\text { saison }\end{array}$ & $\begin{array}{l}\text { - Semailles mil- } \\
\text { maïs } \\
\text { - Travaux } \\
\text { d'entretien } \\
\text { (sarclo-buttage) }\end{array}$ & $\begin{array}{l}\text { Premières } \\
\text { récoltes en fin de } \\
\text { saison }\end{array}$ & $\begin{array}{l}\text { - Maturité des } \\
\text { céréales : récolte } \\
\text { d'arachide, du } \\
\text { maïs sec } \\
\text { - Stockage } \\
\text { - Résidus pour la } \\
\text { période sèche }\end{array}$ \\
\hline $\begin{array}{l}\text { Activités } \\
\text { des éleveurs } \\
\text { étrangers }\end{array}$ & $\begin{array}{l}\text { Arrivée des } \\
\text { transhumants }\end{array}$ & $\begin{array}{l}\text { - Arrivée massive } \\
\text { des transhumants } \\
\text { - Usage des } \\
\text { ressources de la } \\
\text { commune }\end{array}$ & $\begin{array}{l}\text { - Exploitation des } \\
\text { ressources de la } \\
\text { commune } \\
\text { - Retour des } \\
\text { transhumants }\end{array}$ & $\begin{array}{l}\text { Remontée } \\
\text { des éleveurs } \\
\text { nationaux et } \\
\text { étrangers }\end{array}$ & $\begin{array}{l}\text { Remontée ou } \\
\text { retour progressif } \\
\text { des éleveurs } \\
\text { nationaux et } \\
\text { étrangers }\end{array}$ & $\begin{array}{l}\text { Retour progressif } \\
\text { des éleveurs } \\
\text { nationaux }\end{array}$ & $\begin{array}{l}\text { Début d'arrivée } \\
\text { des éleveurs } \\
\text { étrangers dans la } \\
\text { commune }\end{array}$ \\
\hline
\end{tabular}

- l'accueil des populations des zones traversées et de la commune (8\% des éleveurs).

\section{Couloirs d'entrées et axes de transhumance}

Sur la base des points de passage géoréférencés, on peut définir les axes empruntés par les transhumants. Il convient cependant de noter que les points d'entrée dans la commune restent très diffus.

Les mouvements des troupeaux variaient selon la période et les lieux de provenance et de destination. Ainsi, les éleveurs de bovins en transhumance à Djidja étaient originaires du Nigeria, du Niger, du Burkina Faso et du Togo. La taille de leur troupeau a varié d'une année à l'autre les cinq dernières années. Les estimations des techniciens d'élevage ayant en charge la gestion de la transhumance à Djidja ont montré une évolution positive, allant de 13126 têtes de bovins en 2012 à 16800 têtes en 2016 (figure 7).
Deux mouvements de transhumance ont été observés à Djidja au cours d'une année. Au début de la saison pluvieuse, les troupeaux transhumants ont quitté l'ouest du Bénin (Togo et Burkina Faso) et se sont dirigés vers l'est (Bohicon et Nigeria). Le mouvement inverse a été observé au début de la saison sèche. Ceux qui se rendaient au Nigeria étaient en quête de pâturage, alors que ceux qui allaient à Bohicon étaient des commerçants de bétail. Ils s'y rendaient pour vendre des animaux dans les marchés à bestiaux de Bohicon (Gnidjazoun, Zakpo, Saclo et Avokanzoun) et d'Abomey (Djègbé). La figure 5 montre plusieurs axes de transhumance.

Selon les résultats des enquêtes, l'axe de transhumance pendant la saison sèche était choisi le plus souvent par l'éleveur propriétaire après avoir reçu des conseils et des informations auprès du garso ou du saliki. Ce sont des éclaireurs qui fournissent des informations 

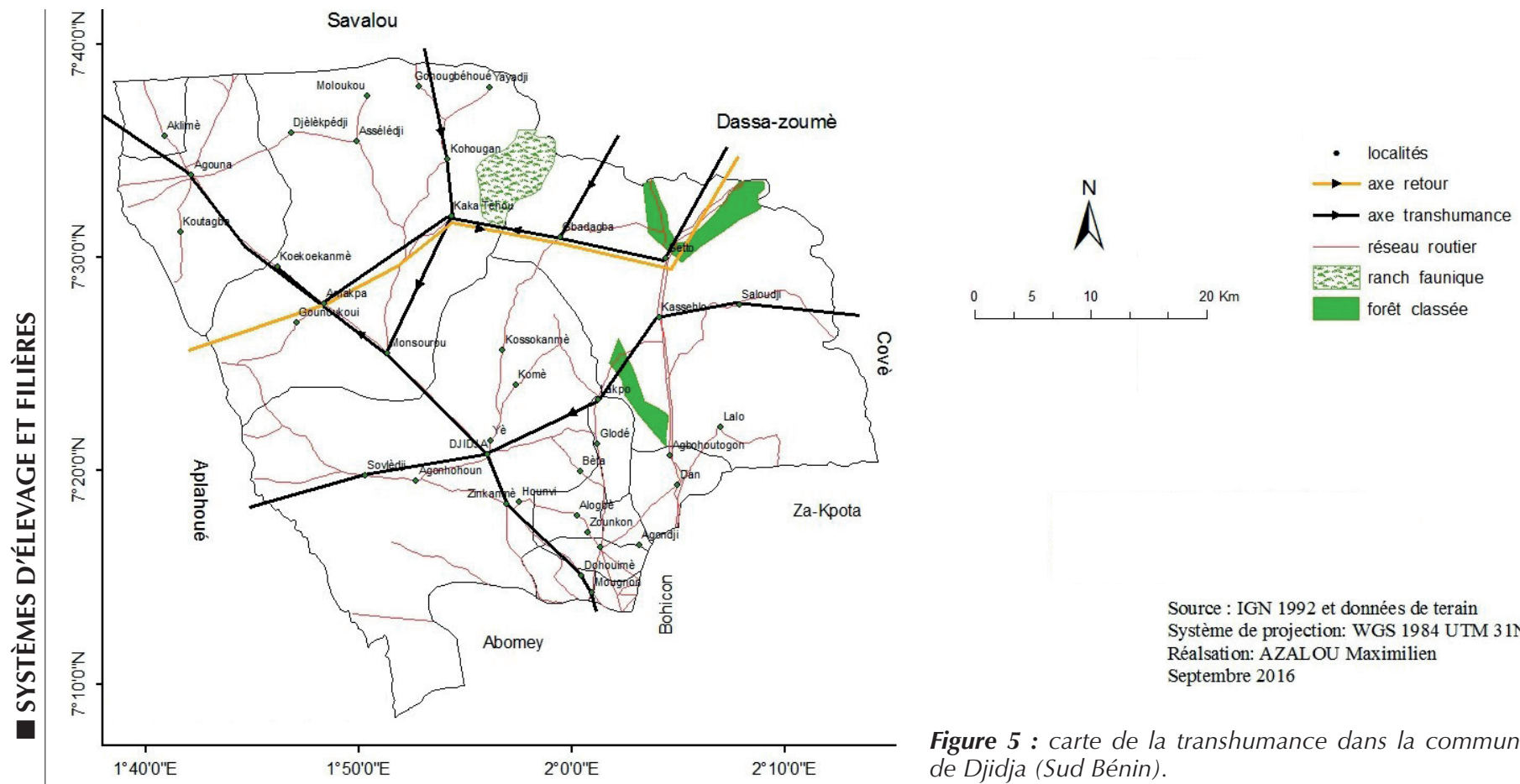

Source : IGN 1992 et données de terain Système de projection: WGS 1984 UTM $31 \mathrm{~N}$ Réalsation: AZALOU Maximilien Septembre 2016

Figure 5 : carte de la transhumance dans la commune de Djidja (Sud Bénin).

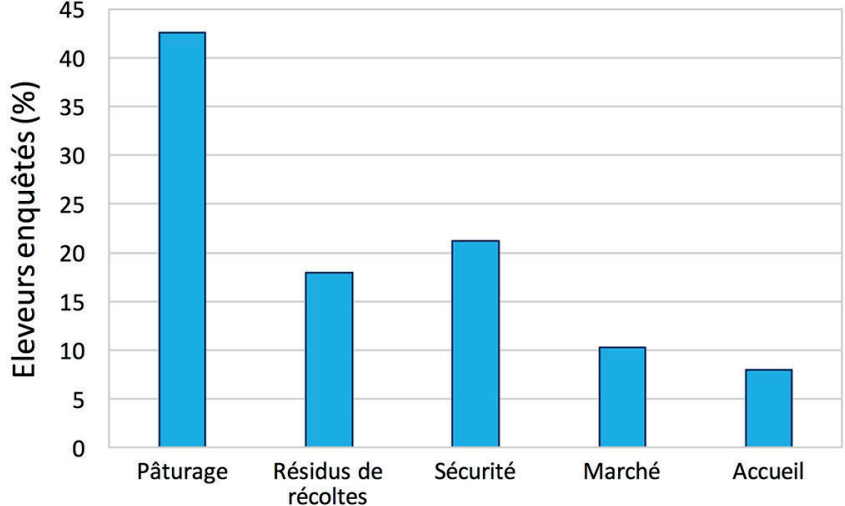

Figure 6 : critères de choix des éleveurs concernant l'itinéraire de mouvement et le campement de nuit (commune de Djidja, Sud Bénin).

aux propriétaires et bouviers en transhumance sur la qualité des ressources, la pluviométrie ainsi que les axes à emprunter pour faciliter leur séjour et la transhumance. Ils interviennent également dans la gestion des conflits entre éleveurs et autres acteurs de la transhumance. Toutefois, la réalité du terrain peut amener le bouvier transhumant à changer le parcours choisi par le propriétaire, notamment la disponibilité des ressources pastorales sur l'axe. Les itinéraires peuvent également changer selon certains éleveurs, en raison de conflits qui causent la mort de plusieurs de leurs animaux, ou du paiement d'amendes.

\section{DISCUSSION}

\section{Dynamique des troupeaux transhumants au sud du Bénin}

Pendant la saison sèche, les éleveurs transhumants se déplacent sur de très longues distances, dans une direction générale sud, à la recherche de pâturages et de points d'eau naturels. Les éleveurs transhumants négocient l'accessibilité aux ressources pastorales, notamment les résidus de

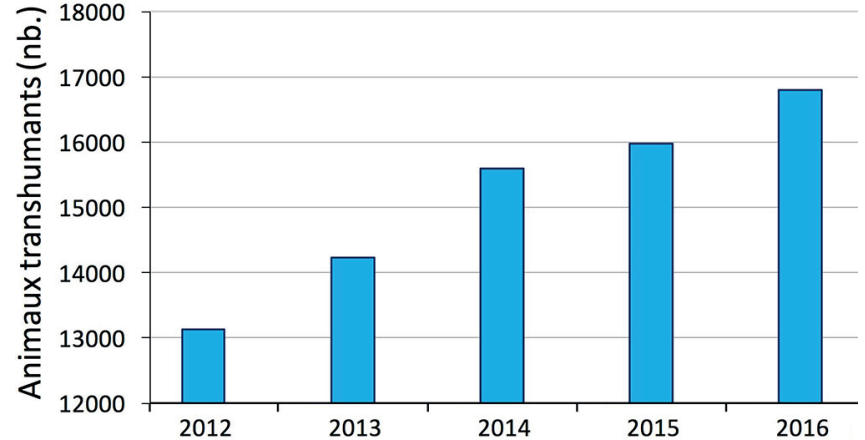

Figure 7 : évolution des effectifs d'animaux étrangers transhumant dans la commune de Djidja (Sud Bénin) de 2012 à 2016 selon les techniciens d'élevage en charge de la gestion de la transhumance dans la commune.

culture, avec les producteurs locaux (Gonin et Tallet, 2012). Les éleveurs des communes voisines de Djidja y transhument avec leurs troupeaux, mais comme ils sont liés par des rapports de bon voisinage au regard des activités menées de part et d'autre, tous sont légitimes à utiliser les ressources de proximité. Par contre, les éleveurs peuls en transhumance font face à de nombreuses accusations lorsque leur troupeau pénètre dans les champs voisins occasionnant de nombreux dégâts. Les conflits et tensions existent en permanence entre les éleveurs transhumants et les agriculteurs lors des déplacements. Gangneron (2013) rapporte des observations similaires et souligne que les accusations portent sur les injustices perçues par les éleveurs locaux d'un partage des ressources qui leur est imposé. Les espaces pastoraux à Djidja se réduisent continuellement en raison notamment de l'augmentation des superficies cultivées et des conséquences liées au changement climatique. Dans le même temps, les effectifs d'animaux, comme les troupeaux bovins en transhumance, augmentent sensiblement.

Les déplacements des troupeaux sont réglés par des savoirs techniques locaux basés sur un découpage spécifique du temps et de l'espace en unités pastorales adaptées à chaque contexte local. Les éleveurs peuls découpent l'année en sept périodes auxquelles ils se réfèrent toujours 
lorsqu'ils parlent de la conduite de leur troupeau. Ce résultat est similaire à celui rapporté par Dongmo et al. (2009) sur la gestion du territoire par les éleveurs peuls en zone soudano-sahélienne au Cameroun et au Burkina Faso.

Le découpage de l'année en sept périodes dans cette étude diffère de celui évoqué par Assani (2017), Djenontin et al. (2012), Dongmo (2009), et Vall et al. (2009) qui ont identifié cinq périodes pastorales : Ceedu, Seeto, Nduungu, Djaamdè et Dabuundè. Le caractère pastoral du calendrier à Djidja est important car il permet à léleveur de connaître la disponibilité et la qualité des fourrages et de l'eau d'abreuvement, indispensables pour assurer l'entretien ou la production du troupeau bovin, ou pour la subdivision du territoire pastoral. Ainsi, Djidja reste le grenier du département du Zou en raison de ses fortes potentialités agricoles. Cette particularité serait liée à la forte descente des éleveurs transhumants en période sèche pour l'exploitation des ressources pastorales, notamment les résidus de cultures et pâturages inexploités jusqu'alors. La référence au calendrier pastoral est à relier à la gestion des espaces pastoraux et culturaux, et à la gestion des relations avec les autres acteurs du territoire pastoral (Djenontin et al., 2012 ; Vall et al., 2009).

La petite transhumance à Djidja a lieu pendant les périodes de $N d u u n g u$ Mawdo, N. Pamarel et Jahol, coïncidant avec les activités agricoles (tableau II). Elle permet à l'éleveur d'éviter la dégradation des champs par les animaux tout en assurant les productions du troupeau (Djenontin et al., 2004 ; Sokemawu, 2011). Ce type de transhumance impacte principalement les éleveurs locaux qui ont des effectifs bovins importants qu'il s'agit de déplacer du campement aux zones de pâturages. L'eau influence le déplacement des Peuls, ainsi les mouvements de transhumance sont liés à l'abondance des pluies (Sokemawu, 2011).

La superposition du calendrier pastoral au diagramme climatique est similaire à celles obtenues par Assani (2017), Djenontin et al. (2012), et Dongmo (2009) qui retrouvent les trois périodes principales du groupe peul que sont Nduungu Mawdo et N. Pamarel, Dabuundè, et Cheedu dans la plupart des calendriers proposés. Dans notre étude, une période de Djaamdè s'intercale entre les périodes de Nduungu Mawdo et $N$. Pamarel qui correspondent à la première récolte. Cette période (Djaamdè) n'a pas été prise en compte par les éleveurs lors de la réalisation du calendrier pastoral car elle n'influence pas la petite transhumance. Cette particularité peut être liée aux caractéristiques climatiques de la zone qui comprend quatre saisons, dont deux saisons pluvieuses et deux saisons sèches.

\section{Itinéraires de transhumance, source de conflits}

Les parcours des animaux suivent une chronologie saisonnière précise. En saison sèche, les pasteurs peuls des pays ou des communes voisines de la zone d'étude conduisent leur troupeau à la recherche de ressources pastorales, ce qui suscite des querelles : «L'exploitation conjuguée par les éleveurs locaux et par ces grands pasteurs conduit à l'épuisement précoce des pâturages » (Gangneron, 2013). Djidja constitue une zone d'accueil des transhumants pendant la saison sèche et une zone de refuge pour les éleveurs pendant la saison pluvieuse. Les transhumants transfrontaliers et nationaux font leur entrée dans la commune par les arrondissements de Setto et Dan. Les axes de transhumance et les zones d'accueil choisis sont fonction de la disponibilité en cours d'eau permanents (fleuves Zou et Couffo) et en pâturages, en l'occurrence ligneux et résidus de récoltes. Le même constat a été fait par Nori (2007), et Djenontin et al. (2009) qui soulignent que l'exploitation cyclique des pâturages naturels et leur extension progressive révèlent la variabilité de la disponibilité en fourrage, et aussi celle des itinéraires dans le temps et dans l'espace. Les zones d'accueil très parcourues disposent de pâturages très appétés et dont la qualité s'est maintenue depuis des décennies, principalement grâce à la mobilité du cheptel (Leclerc et Sy, 2011). Plusieurs explications sont données par les pasteurs pour motiver le déplacement de leur campement principal vers d'autres zones d'accueil : la recherche de pâturages de qualité, d'eau gratuite ou facile d'accès, de marchés, de meilleures conditions sanitaires, d'éléments minéraux et d'espace de pratique pastorale (Sy, 2011).

La présence de multiples itinéraires de transhumance choisis en fonction de la disponibilité en ressources pastorales a des conséquences sur ces ressources et la population. Pendant la saison sèche, la disparition de la strate herbacée contenant les principales graminées fourragères des animaux rend difficile l'alimentation de ceux-ci (Assani, 2017). L'augmentation des effectifs d'animaux au moment où les ressources pastorales fluctuent ou diminuent explique, entre autres, l'accroissement des risques de conflits entre transhumants et sédentaires. Ces risques se traduisent souvent en conflits. La compétition entre groupes qui revendiquent les mêmes droits sur des espaces qui soutiennent leur système de production s'aggrave et se complique davantage lorsque des transhumants étrangers ajoutent de la pression à la charge pastorale déjà lourde (Sy, 2011). Les conflits d'usage de l'espace sont caractérisés par une forte diversité d'expression en fonction des activités, des usages autour desquels ils prennent naissance, des territoires où ils se localisent, ainsi que des caractéristiques des acteurs (Torre et al., 2010). A Djidja, le mode de règlement des conflits mobilise différents niveaux d'instances. Les niveaux retenus sont le règlement coutumier, la justice et la conciliation. « Si tous les conflits n'éclatent pas, il faut souligner l'importance de tous les processus peu ou pas visibles par lesquels des acteurs en concurrence élaborent des compromis, renoncent à l'escalade d'un conflit possible et arrivent à cohabiter sur le mode de la tension et/ou négociation, plutôt que celui de l'affrontement violent » (Kossoumna, 2012).

Dans ce contexte, les éleveurs transhumants, soucieux de la survie de leurs animaux, émondent fortement certains ligneux fourragers tels que Pterocarpus erinaceus, Khaya senegalensis, Afzelia africana (Houinato et Sinsin, 2000 ; Kagoné et al., 2006). L'émondage d'arbres fourragers apporte un complément de nourriture aux animaux pendant cette période de soudure alimentaire. La forêt classée de Dan joue également ce dernier rôle car elle regorge d'herbacées bien appétées par les bovins comme Sporobolus pyramidalis, Andropogon gayanus, et des ligneux très appétés comme Pterocarpus erinaceus, Terminalia glaucescens, Khaya senegalensis et Afzelia africana. Cette affluence vers les forêts classées a été signalée dans plusieurs travaux (Assani, 2017 ; Sinsin, 1998 ; Yemadjè, 2004). De plus, au niveau des points d'abreuvement des animaux, le comblement et la pollution des cours d'eau sont souvent observés. Ainsi, les défécations dans les cours d'eau sont sources de maladies aussi bien pour les animaux et leurs conducteurs, que pour les populations vivant en aval (Assani, 2017 ; Bouraïma, 2007).

\section{Périodes de transhumance et périodes pastorales : une alternance?}

Tous les éleveurs rencontrés à Djidja ont distingué une petite transhumance (pendant la grande et la petite saison pluvieuse) et une grande transhumance (pendant la saison sèche) en prenant en compte les lieux et la durée de la transhumance. Chacune de ces périodes de transhumance correspond à une période pastorale marquée par des activités pastorales. Ainsi, le départ et le retour des éleveurs pratiquant la grande transhumance à Djidja correspondent respectivement à la période de saison sèche froide (Dabuundè) et de début des saisons des pluies (Seeto). Pendant la saison sèche chaude (Cheedu), les transhumants sont dans les zones d'accueil de la grande transhumance. La petite transhumance ou transhumance de saison pluvieuse se déroule pendant Nduungu Mawdo, N. Pamarel et Jahol avec le retour des troupeaux dans leur zone d'attache à la saison des récoltes des cultures (Djaamdè). Nos résultats sont similaires à ceux de Assani (2017), et Djenontin et al. (2012) qui ont également fait un rapprochement entre le déplacement des troupeaux et le calendrier pastoral lors de leur étude réalisée respectivement sur les troupeaux 
transhumants fréquentant la forêt classée de l'Alibori supérieur et les éleveurs du Nord Bénin. La gestion des espaces pastoraux et des troupeaux est fonction des périodes de l'année permettant d'organiser au mieux les activités pastorales (Djenontin et al., 2012).

En réalité, les zones disposant de peu de résidus de récolte sont celles que les troupeaux de bovins en transhumance quittent le plus tôt. La disponibilité des résidus retarderait les dates de départ en transhumance. Suivant les saisons, les périodes de déplacement peuvent varier (Kiema et al., 2014). Cette forme de gestion des ressources naturelles et des troupeaux de bovins selon les périodes est dynamique et très flexible. Une analyse de l'évolution de la gestion temporelle du troupeau montre une hiérarchisation des fonctions du troupeau suivant les saisons en fonction de la disponibilité des ressources du terroir autant à l'échelle du terroir villageois que de l'exploitation agricole (Djenontin et al., 2004).

\section{CONCLUSION}

L'étude a permis de décrire la gestion des ressources naturelles dans la zone d'étude. Les éleveurs transhumants nationaux et étrangers ont développé des stratégies pour l'exploitation des pâturages naturels et pour le maintien des productions de leur troupeau. Le calendrier pastoral, en dehors des sept subdivisions générales, a une spécificité qui est liée à la zone d'étude, notamment la forte production céréalière. Elle permet à l'éleveur d'orienter ses déplacements pour une gestion efficace de l'espace pastoral et de son troupeau au sud du Bénin. L'étude des itinéraires empruntés par les éleveurs fait ressortir six principales directions animées par les axes de transhumance vers des sites d'accueil qui sont choisis en fonction de la disponibilité des ressources pastorales. Ainsi, dans leurs rapports sociaux avec l'espace, les pasteurs construisent et consolident en permanence des structures sociospatiales articulées autour de réseaux diffus et flexibles, entre et autour des zones de résidence, de transit et d'accueil.

\section{Remerciements}

Les auteurs expriment leur gratitude aux éleveurs transhumants de la commune de Djidja pour leur franche et précieuse collaboration pendant cette étude.

\section{Déclaration des contributions des auteurs}

MA, AAS et BGCA ont participé à la conception et à la planification de l'étude. MA, JSA et HSSW ont collecté les données sur le terrain et les ont dépouillées et saisies. MA et BGCA ont rédigé la première version du manuscrit. MNB et IAT ont aussi participé à la conception de l'étude. MA, AAS et MNB ont effectué les analyses statistiques et interprété les résultats d'analyse. MNB et IAT ont participé à la révision critique du manuscrit et ont donné leur accord sur la version finale à publier. Tous les auteurs autorisent la soumission de la version finale en vue de sa publication dans Rev. Elev. Med. Vet. Pays Trop.

\section{REFERENCES}

Apostolidis T., 2006. Représentations sociales et triangulation : une application en psychologie sociale de la santé. Psicol. Teor. Pesqui., 22 (2) : 211-226, doi : 10.1590/\$0102-37722006000200011

Ardilly P., 2006. Les techniques de sondage, $2^{\mathrm{e}}$ édn. Technip, Paris, France, $688 \mathrm{p}$.

Assani S.A., 2017. Transhumance dans la forêt classée de l'Alibori supérieur au nord du Bénin : acteurs, pratiques d'élevage et modèle conceptuel de I'exploitation des ressources pastorales. Thèse Doct., Ecole doctorale des sciences agronomiques et de l'eau, Université de Parakou, Bénin, 203 p.

Azalou M., 2016. Caractérisation des troupeaux bovins en transhumance dans les régions du sud Bénin : cas de la commune de Djidja. Mém. Master Prof., Faculté d'agronomie, Université de Parakou, Bénin, 99 p.
Bardin L., 1998. L'analyse de contenu, ge édn. Presses universitaires de France, Paris, 291 p.

Bouraïma A., 2007. Etat des lieux quantitatif et spatialisé de la transhumance dans la commune de Gogounou. Mém. ingénieur agronome, Faculté d'agronomie, Université de Parakou, Bénin, 88 p.

Dagnelie P., 1998. Statistique théorique et appliquée, vol. 2. De Boeck et Larcier, Paris, France, 659 p.

de Jode, H., 2010. Modern and mobile: the future of livestock production in Africa's drylands. IED and SOS Sahel, 92 p., pubs.iied.org/pdfs/12565IIED. pdf

Djenontin J.A., Amidou M., Baco N.M., 2004. Diagnostic gestion du troupeau : gestion des ressources pastorales dans les départements de I'Alibori et du Borgou au nord Bénin. Bull. Rech. Agron. 43 : 30-45

Djenontin J.A., Houinato M.R., Toutain B., Sinsin B.A., 2009. Pratiques et stratégies des éleveurs face à la réduction de l'offre fourragère au Nord-Est du Bénin. Sécheresse, 20 : 346-53

Djenontin J.A., Madjidou O., Houinato M.R., Mensah G.A., Sinsin B.A., 2012. Le calendrier pastoral en élevage extensif dans le nord-est du Bénin : un outil de gestion du cheptel bovin de l'exploitation. Sécheresse, 23 : 261-70, doi : $10.1684 /$ sec.2012.0350

Dongmo A.L., 2009. Territoire, troupeaux et biomasse : enjeux de gestion pour un usage durable des ressources au Nord Cameroun. Agro Paris Tech, France, $275 \mathrm{p}$.

Dongmo A.L., Vall E., Diallo M.A., Dugue P., Dehoux J.P., 2009. Gestion du territoire par les éleveurs peuls en zone soudano-sahélienne: Apports des savoirs locaux dans l'analyse des pratiques au Cameroun et au Burkina Faso. Rencontres Rech. Rumin.

Duteurtre G., Kamil H., Le Masson A., 2002. Etude sur les sociétés pastorales au Tchad. Rapport de synthèse. Cirad-Emvt/NSF/LRVZ, 84 p.

Floquet A., Nansi, J., 2005. Plus de place en ville pour les bœufs : La filière bovine face à I'expansion urbaine à Abomey et Bohicon (Bénin). Cebedes, Ecocité, Cotonou, Bénin, 64 p. (Document de travail ; 4)

Gangneron F., 2013. Ressources pastorales et territorialité chez les agroéleveurs sahéliens du Gourma des buttes. VertigO, 13 (3) : doi : 10.4000/ vertigo. 14427

Gonin A., Tallet B., 2012. Changements spatiaux et pratiques pastorales : les nouvelles voies de la transhumance dans l'ouest du Burkina Faso. Cah. Agric., 21 : 448-454

Guidibi M., Akomagni L.A., 2006. Monographie de la commune de Djidja (rapport d'étude). Mission de décentralisation / PRODECOM, Bénin

Harchies M., Binot A., Wolff E., 2007. Impacts mutuels de la conservation et de l'élevage transhumant sur l'occupation des sols et les ressources environnementales : une étude de cas camerounaise. VertigO (hors sér. 4)

Hiya Maidaya M., Andres L., Yamba B., Lebailly P., 2016. Mobilité pastorale au Sahel et en Afrique de l'Ouest : essai de synthèse. In : Journées scientifiques, Université Abdou Moumouni, Niamey, Niger, 20-22 janv. 2016

Houinato M., Sinsin B., 2000. La pression agropastorale sur la zone riveraine de la réserve de biosphère de la Pendjari. Tropicultura, 18 : 112-117

Kagoné H., Toutain B., Dulieu D., Houinato M., Boureima A., Nocker U., 2006. Pastoralisme et aires protégées en Afrique de l'Ouest : du conflit à la gestion concertée de la transhumance transfrontalière dans la région du Parc régional W (Bénin, Burkina Faso, Niger). Bull. Anim. Health Prod. Afr., 54: 43-52, doi: 10.4314/bahpa.v54i1.32729

Kiema A., Tontibomma G.B., Zampaligré N., 2014. Transhumance et gestion des ressources naturelles au Sahel : contraintes et perspectives face aux mutations des systèmes de productions pastorales. VertigO, 14 (3) : doi : 10.4000/vertigo.15404

Kossoumna L.N., 2012. Sédentarisation des éleveurs transhumants dans le Nord du Cameroun : évolution des conflits ruraux et de leurs modes de résolutions. Afr. Popul. Stud., 26 : 113-132

Leclerc G., Sy O., 2011. Des indicateurs spatialisés des transhumances pastorales au Ferlo. Cybergeo (532) : 10.4000/cybergeo.23661

Lesse P., 2016. Gestion et modélisation de la dynamique des parcours de transhumance dans un contexte de variabilités climatiques au NordEst du Bénin. Thèse Doct., Université d'Abomey-Calavi, Faculté des sciences agronomiques, Bénin, 299 p. 
Marty A., Bonnet B., Guibert B., 2006. La mobilité pastorale et sa viabilité. Entre atouts et défis. Iram, Paris, France, 4 p. (Note thématique ; 3)

Nori M., 2007. La mobilité pastorale : une histoire à réécrire. Cours modulaire sur le pastoralisme au Master PARC (productions animales en régions chaudes). Cirad, Montpellier, France, $5 \mathrm{p}$.

Schönegg G., Martel P., Sano B., Noufou S., 2006. Les conflits liés à la transhumance transfrontalière entre le Niger, le Burkina Faso et le Bénin. DED, Bonn, Allemagne

Sinsin B.A., 1998. Transhumance et Pastoralisme. In : Les aires protégées d'Afrique francophone (éd. Sournia G.). Monza, Paris, France, 26-31

Sokemawu K., 2011. Déterminants, incidences et contraintes du pastoralisme transhumant dans la région des savanes au Togo. Rev. Geogr. Trop. Environ. (1) : 44-59

Sy O., 2011. Dynamique de la transhumance et perspectives d'un développement intégré dans les régions agro-sylvo-pastorales du Ferlo (Sénégal). Rev. Geogr. Lab. Leïdi : 125-139
Torre A., Melot R., Bossuet L., Cadoret A., Caron A., Darly S., Jeanneaux P., et al., 2010. Comment évaluer et mesurer la conflictualité liée aux usages de l'espace ? Eléments de méthode et de repérage. VertigO, 10 (1) : doi 10.4000 /vertigo.9590

Toutain B., Marty A., Bourgeot A., Ickowicz A., Lhoste P., 2012. Pastoralisme en zone sèche. Le cas de l'Afrique subsaharienne. Agropolis international, Montpellier, France, 2 p. (CSFD dossier thématique ; 9)

Vall E., Blanchard M., Diallo M.A., Dongmo A.L., Bayala I., 2009. Savoirs techniques locaux, sources d'innovations ? Production de savoirs actionnables dans une démarche de recherche-action en partenariat. In : Savanes africaines en développement : Innover pour durer. Cirad, Montpellier, France, $14 \mathrm{p}$.

Vissin E.W., 2015. Gestion des risques hydro-climatiques et développement économique durable dans le bassin du Zou. J. Rech. Sci. Univ. Lomé, 17 (3) : 191-213

Yemadjè A.A.S., 2004. Transhumance dans la région des Monts-Kouffè au Bénin. Mém. maîtrise, Université d’Abomey-Calavi, Flash, Bénin, 96 p.

\section{Summary}

Azalou M., Assani Seidou A., Assogba B.G.C., Adjassin J.S., Sanni Worogo H.S., Baco M.N., Alkoiret Traoré I. Pastoral calendar and transhumance map of herders using pastoral resources in Djidja Commune in Southern Benin

Djidja is one of the largest agricultural-producing communes of the department of Zou in Southern Benin. Because of its fodder and water resources, it has become a destination for transhumant livestock farmers. Movements of transhumant herders continue to expand, as does the length of stays in this reception area. The overall objective of the study was to develop the pastoral calendar and transhumance map of the herders staying in this district. Semi-structured interviews were conducted with 300 transhumance stakeholders. The survey showed that transhumance in this district was mainly caused by the search for fodder and water resources (78.7\%). Seven periods (Seeto, Nduungu Mawdo, Nduungu Pamarel, Jahol, Djaamdè, Dabuundè and Cheedu) were identified in the calendar of transhumant herders in this area with the particularity of two Nduungu periods (rainy season). This particularity is related to the climatic data on the area, which includes four seasons, i.e. two rainy and two dry. The pastoral calendar, itinerary followed and stay periods depended on the available pastoral resources in the place of origin, during herd movements, and in reception areas. Thus, a strong knowledge of transhumant routes, entry and exit points, and settling-down periods for transhumant herders will serve as tools for decision makers in the sustainable management of transhumance and pastoral resources in Southern Benin.

Keywords: cattle, rearing system, calendar, transhumance, crop residues, pastoralism, Benin

\section{Resumen}

Azalou M., Assani Seidou A., Assogba B.G.C., Adjassin J.S., Sanni Worogo H.S., Baco M.N., Alkoiret Traoré I. Calendario pastoral y mapa de trashumancia de pastores que utilizan recursos pastorales en la comuna de Djidja en el sur de Benin

Djidja es uno de los municipios más grandes de producción agrícola del departamento de Zou, en el sur de Benin. Debido a su forraje y recursos hídricos, se ha convertido en un destino para los ganaderos trashumantes. Los movimientos de los pastores trashumantes continúan expandiéndose, al igual que la duración de las estancias en esta área de recepción. El objetivo general del estudio fue desarrollar el calendario pastoral y el mapa de trashumancia de los pastores que se alojan en este distrito. Se realizaron entrevistas semiestructuradas con 300 participantes en trashumancia. La encuesta mostró que la trashumancia en este distrito se debió principalmente a la búsqueda de forraje y recursos hídricos $(78,7 \%)$. Se identificaron siete períodos (Seeto, Nduungu Mawdo, Nduungu Pamarel, Jahol, Djaamdè, Dabuundè y Cheedu) en el calendario de pastores trashumantes en el área, con la particularidad de dos períodos de Nduungu (temporada de lluvias). Esta particularidad está relacionada con los datos climáticos del área, que incluye cuatro estaciones, es decir, dos lluviosas y dos secas. El calendario pastoral, el itinerario seguido y los períodos de permanencia dependieron de los recursos pastorales disponibles en el lugar de origen, durante los movimientos de la manada y en las zonas de recepción. Por lo tanto, un sólido conocimiento de las rutas trashumantes, de los puntos de entrada y salida, y de los períodos de permanencia de los pastores trashumantes servirán como herramientas para los diversos responsables de la toma de decisiones en la gestión sostenible de la trashumancia y de los recursos pastorales en el sur de Benin.

Palabras clave: ganado bovino, sistemas de cría, calendario, trashumancia, residuos de cosechas, pastoralismo, Benin 
